\title{
Microstructures in phase inversion membranes. Part 2. The role of a polymeric additive*
}

\author{
R.M. Boom, I.M. Wienk, Th. van den Boomgaard and C A. Smolders \\ Department of Chemical Technology, University of Twente, $P O$ Box 217, 7500 AE Enschede (The Netherlands)
}

(Received July 31, 191, accepted in revised form April 16, 1992)

\begin{abstract}
Membranes were prepared from a casting solution of a water-soluble polymer, poly (vinyl pyrrolidone) (PVP), and a membrane forming polymer, poly (ether sulfone), in 1-methyl-2-pyrrolidone (NMP) as solvent by immersing them in mixtures of water and NMP It was found that the addition of PVP to the ternary system suppresses the formation of macrovoids in the sub-layer, whlle the ultrafiltration-type top-layer consists of a closely packed layer of nodules Using a model for mass transfer in this quaternary system, it is possible to explain the effects of the additive on macrovoid formation Strong indications are found that the appearance of a nodular structure in the top-layer follows a mechanism of spinodal decomposition during the very early stages of the immersion step
\end{abstract}

Keywords membrane preparation and structure, theory, thermodynamics, macrovoids, phase-inversion membranes

\section{Introduction}

Phase inversion is the most important process to prepare symmetric or asymmetric membranes Because of the significance of immersion precipitation (phase inversion), the mechanism of formation of these membranes has been the subject of extensive investigation [1,2] In recent years, Reuvers et al [1] developed a model for the description of mass transfer during the immersion step Two types of de-

Correspondence to R M Boom, Department of Chemical Technology, University of Twente, P O Box 217, 7500 AE Enschede, The Netherlands

*Paper presented at the Int Symp on "Progress in Membrane Science and Technology", Enschede, The Netherlands, June 25-28, 1991 mixing occurring during immersion precipitation followed from this model. instantaneous demixıng and delayed demixıng. Taking this model of mass transfer in combination with liquid-liquid phase separation, effects of variations in the composition of the casting solution and the coagulation bath could be explained

On the basis of the distinction between delayed and instantaneous demixing, Reuvers et al proposed a mechanism for the formation of large fingerlike cavities (macrovoids), often occurring in the sub-layer of immersion precipitation membranes (see preceding Part 1). Although this model is strictly valid only for ternary systems consisting of a membrane forming polymer, a solvent and a non-solvent for the polymer, some effects of the addition of a fourth 
component to the system can be explained with it.

In order to obtain an optimal membrane structure, an additive (a fourth component) is frequently used. Usually, the additive is a weak non-solvent for the polymer, e g. glycerol in a system consisting of polysulfone, DMAc and water, or maleic acid in a system of cellulose acetate, dioxane and water. Such an additive to the casting solution brings the initial composition of the casting solution nearer to the binodal According to the mechanism proposed by Reuvers et al., this decreases the tendency of the solution to form macrovords.

In other systems, polymeric additıves are used Well-known is the addition of poly (vinyl pyrrolıdone) to a system consisting of polysulfone, and a solvent such as DMAc [3]. The most important effects of this type of additive are suppression of macrovolds, improved interconnectivity of the pores and higher porosities in the top-layer and the sub-layer Further, it appears that the top-layers of these membranes have a nodular structure Reuvers' model can not account for the effects of this type of additive.

In this paper we will show some of the effects of the addition of poly (vinyl pyrrolidone) to the system consisting of poly (ether sulfone) and 1methyl-2-pyrrolidone, coagulated with water. In Part A of this paper the mechanısm underlyıng macrovoid formation will be discussed. We will present a model to describe mass transfer in a quaternary system consisting of two miscible polymers in a common solvent precipıtated by a non-solvent for one of the polymers. On the basis of this model, we will come to a mechanism that can explain some of the effects of the polymeric additive In Part B, the occurrence of nodular structures in UF-type top-layers will be discussed Experimental evidence will be given for a possible spinodal mechanism of formation of these nodular structures in toplayers

\section{(A) Macrovoid formation in systems with a macromolecular additive}

\section{Theoretical considerations}

The system we want to investigate consists of four components.

- A non-solvent for the polymer, initially present in the coagulation bath (component 1).

- A solvent for the polymer, initially present in the polymer solution, and in some cases in the coagulation bath (component 2)

- The membrane forming polymer (component 3 ), present only in the polymer solution and in the final membrane

- The macromolecular additive used (component 4).

We assume that the additive is miscible with all components present. A typical model system for this is the system used in our experiments: water (1)-1-methyl-2-pyrrolidone (2)-poly (ether sulfone) (3)-poly (vinyl pyrrolidone) (4)

\section{Thermodynamics}

We have in our system two macromolecular components, both present at high concentrations in the same solution. Typical concentrations in casting solutions are 15 to 20 weight percent membrane forming polymer and 10 to 15 weight percent additive. The two polymers both are far above their overlap concentrations and form entangled, intertwined coils, since we know that the two polymers are well miscible. This implies that we assume that the FloryHuggins interaction parameter between the additive and the membrane forming polymer is low. In the case of poly (ether sulfone) and poly(vinyl pyrrolidone) this interaction parameter has been determined (with high-pressure osmometry) to be lower than zero, which indicates that the two polymers form homogeneous blends at all concentrations.

Phase separation in such a system involves the demixing of the intertwined polymers. In 
equilibrium, the polymeric additive has moved to the membrane forming polymer lean phase This process is considerably slower than the exchange processes of solvent and non-solvent between the casting solution and the coagulation bath occurring directly upon immersion, because the separation of the two polymers involves the movement of one polymer with respect to the other. It is therefore convenient to distinguish two time scales for the first moments of the immersion step.

(1) The shorter time scale is valid for the process of exchange of solvent and non-solvent. On this time scale, the two polymers effectively behave as one component. Transport of the lowmolecular weight components through the polymeric network is possible, transport of the polymers with respect to each other is not possible.

(2) The longer time scale is the time scale at which the two polymers can move relative to each other the polymeric additıve moves into the polymer lean phase

These time scales have different thermodynamic regimes to which they respond Therefore, the phase diagrams of both time scales should be evaluated From a thermodynamic point of view, the short time scale is characterized by the absence of any polymer in the polymer lean phase. It only contains solvent and non-solvent This characteristic feature will be used to determine the thermodynamics on the short time scale During the longer time scale, the macromolecular additive is allowed to be present in the membrane forming polymer lean phase

\section{Equilıbrium calculations}

The basis of the calculations is the FloryHuggins expression for the chemical potential of mixing in a quaternary systems*

$\frac{\Delta G_{\mathrm{m}}}{R T}=n_{1} \ln \phi_{1}+n_{2} \ln \phi_{2}+n_{3} \ln \phi_{3}$

$$
\begin{aligned}
& +n_{4} \ln \phi_{4}+g_{12} n_{1} \phi_{2}+\chi_{13} n_{1} \phi_{3}+g_{14} n_{1} \phi_{4} \\
& +g_{23} n_{2} \phi_{3}+g_{24} n_{2} \phi_{4}+g_{34} n_{3} \phi_{4}
\end{aligned}
$$

All ternary and quaternary interaction parameters are neglected This means that the phase diagrams calculated should only be used in a semı-quantitatıve way

The derivatives of the free enthalpy of $\mathrm{mix}$ ing with respect to the number of moles of each component are the chemical potentials of mixing Phase equilibria are calculated by using the algorithm proposed by Altena and Smolders [4] The following objective function $F$ is mınımızed

$F=\sum_{t=1}^{4}\left(\frac{\mu_{t}^{\mathrm{a}}-\mu_{\imath}^{\mathrm{b}}}{R T}\right)^{2}$

in which the index a belongs to the concentrated phase while the index $b$ is for the diluted phase, and $\phi_{t}$ and $\mu_{\imath}$ denote the volume fraction and the chemical potential of component $l$, respectively. $T$ is the temperature, and $R$ is the gas constant.

As discussed before, it is assumed that in the diluted phase no polymer is present. In all calculations concerning ternary systems, it appears that, excluding the area around the critical point, the polymer is absent in the polymer lean phase (see e.g Altena et al [4]. Since in our quaternary case in the beginning the two polymers cannot move relative to each other, we have an analogous situation. We assume that both polymers do not significantly move into the coagulation bath. Note, however, that component 4 has a driving force to diffuse out Whenever a single component 4 molecule would diffuse out, the diffusion rate in the coagulation bath is so much higher, compare to the outdiffusion from the polymer solution, that it will diffuse quickly away, and will have no influence on the interface situation Therefore, we can assume that in the coagulation bath only components 1 and 2 are present. The relations for the chemical potentials collapse to those for 
a binary system, and the objective function $F$ in eqn (2) consists only of two terms $(l=1,2)$, those for the chemical potentials of the nonsolvent 1 and the solvent 2

\section{Modelling of mass transfer}

To describe mass transfer in the polymer solution and in the coagulation bath, we expand the model developed by Reuvers et al. [1] We will summarize the most important points from this model The continuity equations are in our case the same as the ones Reuvers used:

$\frac{\partial}{\partial t}\left(\frac{\phi_{l}}{\phi_{3}}\right)=\sum_{\substack{\jmath=1 \\ J \neq \imath}}^{4}\left[\frac{\partial}{\partial m}\left(v_{\imath} \phi_{3} L_{\iota} \frac{\partial \mu_{\jmath}}{\partial m}\right)\right]$

while the binary diffusion in the coagulation bath is described by

$\frac{\partial \phi_{\imath}}{\partial t}=\frac{\partial}{\partial y}\left(D_{\imath} \frac{\partial \phi_{2}}{\partial y}\right)-\frac{\partial \phi_{\imath}}{\partial y}\left(J_{1}^{y=0}+J_{2}^{y=0}\right)$

The $J_{i}^{y=0}$ means there the flux $\left(\mathrm{m}-\mathrm{sec}^{-1}\right)$ of component $\imath$ through the boundary layer between polymer solution and coagulation bath The spatial coordinate $m$ in eqn. (3) is described by:

$m=\int_{\xi=0}^{\xi=x} \phi_{3} \mathrm{~d} \xi$

in which $x$ is the distance from the moving interface (between the polymer solution and the coagulation bath) into the polymer solution The spatial coordinate $y$ into the coagulation bath is taken from the (moving) interface. It is assumed that both the coagulation bath and the polymer solution are semi-infinite. The mobility coefficients $L_{\imath}$ can be found with the Stefan-Maxwell approach, which relates the drivung forces (the chemical potentials) to the velocities $\bar{v}_{\imath}$ of the components.

$\frac{\partial \mu_{\imath}}{\partial x}=\sum_{j=1}^{4} c_{J} R_{\imath}\left(\bar{v}_{J}-\bar{v}_{\imath}\right) \quad \imath=1,2,3,4$

Here, $R_{\imath \jmath}$ is the so-called friction coefficient be- tween component $\imath$ and $J, c_{\imath}$ stands for the concentration of component $\imath$ For a detaled description of how the mobility coefficients are related to the Stefan-Maxwell friction coefficients $R_{l]}$ we refer to Reuvers et al [1], who describes this for a ternary system

Our calculations are aimed only at the short time scale It is assumed that the two polymers cannot move with respect to each other. In formula

$\bar{v}_{3}-\bar{v}_{4}=0$

The Stefan-Maxwell equations now simplify to a semi-ternary problem

$$
\begin{aligned}
\frac{\partial \mu_{\imath}}{\partial x}= & c_{1} R_{\imath 1}\left(\bar{v}_{1}-\bar{v}_{\imath}\right)+c_{2} R_{\imath 2}\left(\bar{v}_{2}-\bar{v}_{\imath}\right) \\
& +\left(c_{3} R_{\imath 3}+c_{4} R_{\imath 4}\right)\left(\bar{v}_{3}-\bar{v}_{\imath}\right) \quad l=1,2
\end{aligned}
$$

Equation (8) is used in combination with the continuity equations (eqns. 3 and 4 ) to calculate the composition profiles as a function of time. We assume that the concentrations at the interface between the casting solution and the coagulation bath are constant. This is a reasonable suggestion for the first moments of $1 \mathrm{~m}$ mersion Reuvers et al [1] showed that in this case, the composition profile is only dependent on the variable (a partial Fourier number).

$l=\frac{m}{\sqrt{t}}$

This indicates that the composition profile calculated not only represents the initial composition profile in the top-layer, but also the dependence on time of the compositions at each position in the polymer solution. The composition profiles were calculated in double precision, with the E03PGF routine from the NAGlibrary. Calculatıons were performed accordıng to the following scheme:

(1) Equilibrium conditions are assumed at the interface; the polymer concentration at the interface is estimated as a first guess. 
(2) Composition profiles are calculated both in the polymer solution and in the coagulation bath

(3) The fluxes at both sides of the interface, found from the calculated composition profiles, are compared

(4) The steps 1 to 3 are repeated with varying polymer concentration at the interface, until the flux through the interface of each component is equal at both sides of the interface

\section{Experimental set up}

\section{Materials}

The membrane forming polymer poly (ether sulfone) (Victrex 5200P) was supplied by ICI Molecular weight was $43800 \mathrm{~g} /$ mole as determined by GPC measurements. The additive poly (vinyl pyrrolıdone) (PVP) was purchased from Janssen Chimica. Different types of PVP were used indicated by a K-number, K15 (MW $10,000 \mathrm{~g} / \mathrm{mole}$ ), K30 (MW 40,000 g/mole ), K60 (MW 160,000 g/mole) and K90 (MW 360,000 $\mathrm{g} / \mathrm{mole}$ ). 1-Methyl-2-pyrrolidone (Merck, synthetıc grade) was used as solvent, whlle the non-solvent used was water.

\section{The spinning process}

Hollow fibers were spun by the dry-wet spinnung technique or by a two-bath system The filtered and degassed polymer solution was pumped through a spinneret. In the dry-wet technique the solution leaving the spinneret passes a airgap (the first age) after which it enters into a coagulation bath (the second stage). On the bore side an internal coagulation bath was pumped In case of the two bath system the first and second stage were two different outer coagulation baths. The first bath and the internal coagulation bath were NMP-water mixtures at ambient temperatures. The second stage always was a water bath and its temperature could be varıed. After spinning, residual solvent was removed by flushing with water for two days

\section{Electron microscopy}

Morphology of the membranes was studied with an electron microscope (JEOL JSM T220A) Sample preparation was done as follows. After displacement of water by ethanol and ethanol by hexane the membranes were dried in air. In a sputtering apparatus (Balzers unıon SCD 040) a thin gold-layer was deposited on the membranes.

\section{Results}

\section{Experimental}

The effects of a polymeric additive on the membrane morphology obtained is shown in Fig 1, which gives cross sections through membranes made with varying concentrations of polymeric additıve and varying molecular weights of the additive. It appears that the molecular weight of the polymeric additive is an important parameter Addition of a polymeric additıve of a certain minımum molecular weight results in the absence of macrovolds in the membrane structure. It further appears that a certain minimum concentration of polymeric additive is needed to suppress macrovord formation

\section{Equilibrium calculations}

Phase equilibria were calculated according to the conditions discussed in the theoretical section. The ratio of weight percentages of components 3 and 4 was varied The resulting phase diagrams are shown in Fig 2

Parameters used are based on measurements for the system poly (ether sulfone)-1-methyl2-pyrrolidone-water, by Zeman and Tkacık [5], and measurements performed in our own laboratory. These measurements were done with high-pressure osmometry a PES-NMP solution was brought into contact with NMP via a 
Cuprophan $^{\mathrm{TM}}$ membrane, that swells only slightly in NMP The polymer was found not to permeate through the membrane measurably over a period of at least one week By determining the equilibrium osmotic pressure in the solution cell, the interaction parameters could be determined

It appears from the computations that with increasing content of component 4 (the additive), the binodal shifts to the non-solvent cor-

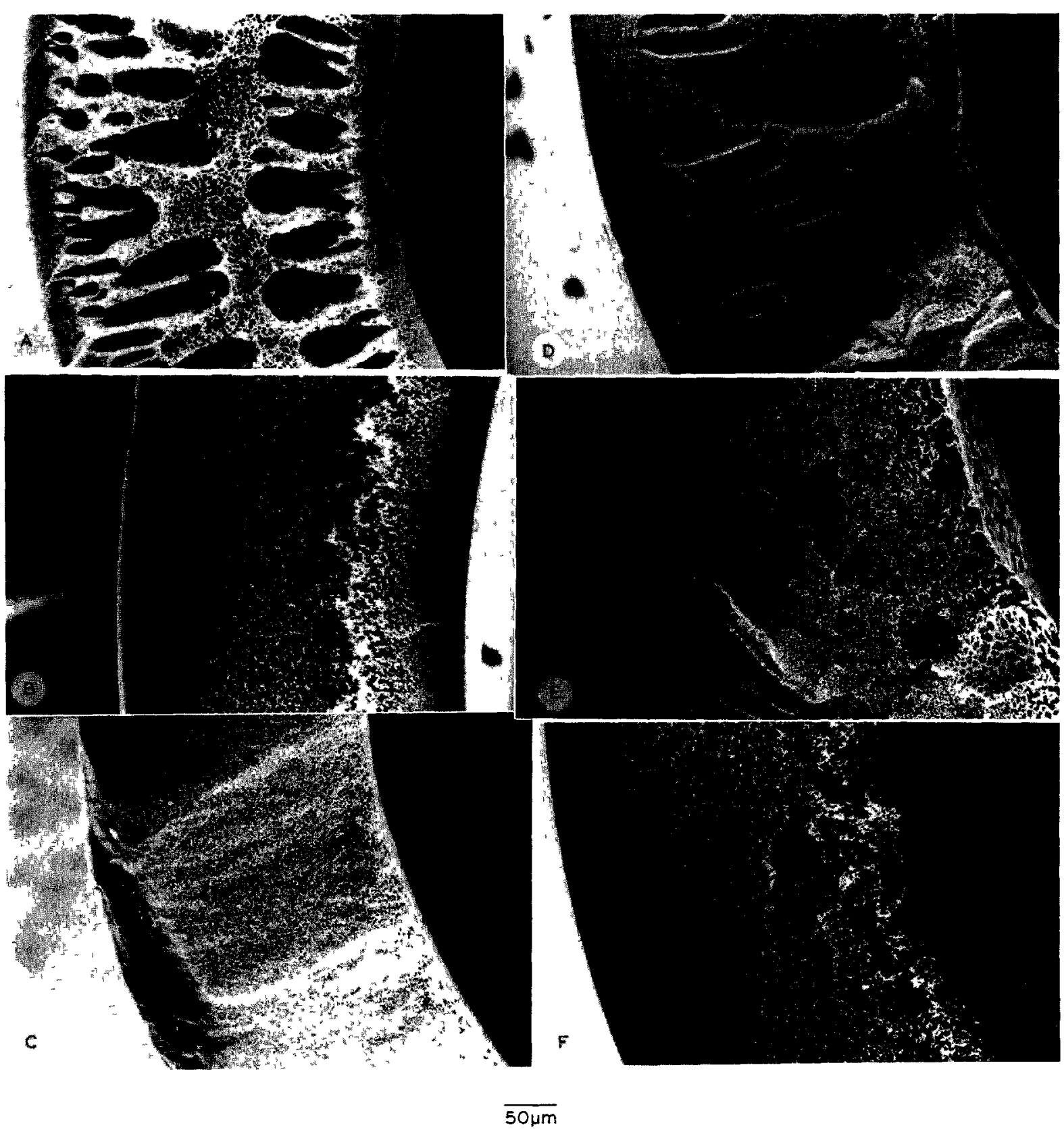




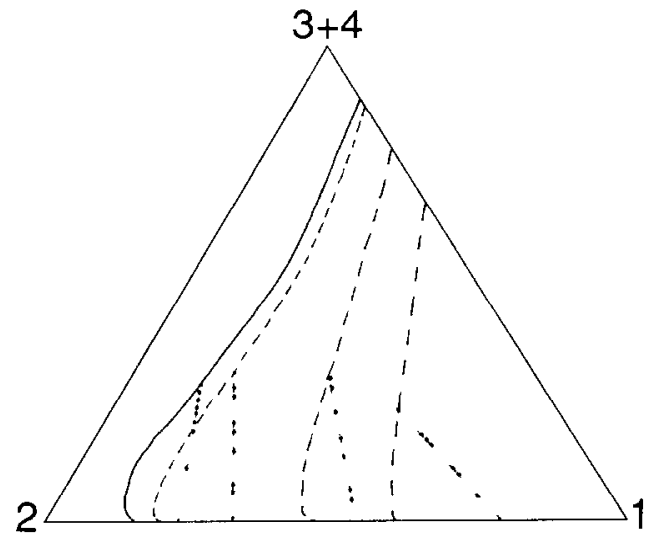

$$
\begin{array}{ll}
-\frac{\varphi_{4}}{\varphi_{3}}=0 & ---\frac{\varphi_{4}}{\varphi_{3}}=01 \\
---\frac{\varphi_{4}}{\varphi_{3}}=05 & ---\frac{\varphi_{4}}{\varphi_{3}}=10
\end{array}
$$

Fig 2 Phase diagrams for the short time scale in systems with a macromolecular additive Parameters used [5] $g_{12}=10, g_{23}=05, \chi_{13}=15, g_{14}=04, g_{24}=05, g_{34}=-10$ Molecular weights (1) $18 \mathrm{~g}$-mole ${ }^{-1}$, (2) $99 \mathrm{~g}$-mole ${ }^{-1}$, (3) $18 \mathrm{~kg}$-mole ${ }^{-1}$, (4) $40 \mathrm{~kg}$-mole ${ }^{-1}$

ner These phase diagrams are only valid as long as the two polymers cannot move relative to each other, i.e as long as polymeric additive is absent in the polymer lean phase.

\section{Mass transfer}

Initial composition profiles were calculated for a system in which the ratio of the weight fractions of the polymeric components is unity It was assumed that the diffusivity of component 4 was equal to the diffusivity of component 3 . In our model system consisting of poly (ether sulfone) (3)-poly(vinyl pyrrolldone) (4)-1methyl-2-pyrrolidone (2), measurements of diffusion coefficients of both polymeric components $(3,4)$ in the solvent (2) justify this assumption These measurements were performed in our laboratory with an analytıcal ultracentrifuge by following a small $\mathrm{im}$ posed gradient in the polymer concentration by means of a Schlieren optical system A typical composition profile calculated is shown in Fig. 3 This profile is only valid for the first instances after immersion

The influence of the concentration of solvent in the coagulation bath has been considered, resulting in the curves in Fig 4 For higher solvent concentrations in the coagulation bath, there is no composition jump at the interface anymore, because the initial composition profile does not reach a binodal composition anymore A gradual transition from the casting solution to the coagulation bath is obtained When a polymeric solution with a composition as discussed is coagulated in a coagulation bath with

Fig 1 Scanning electron microscopic pictures of cross sections through hollow fibers Concentration of poly (ether sulfone) was $20 \mathrm{wt} \%, 5 \mathrm{wt} \%$ water was added to the casting solution In case of $\mathrm{A}, \mathrm{B}$ and $\mathrm{C}$ the indicated coagulation bath is the bore liquid For D, E and F the indicated coagulation bath is the first external stage of the bath, the second stage was a waterbath of $24^{\circ} \mathrm{C}$

\begin{tabular}{lll}
\hline & $\begin{array}{l}\text { Casting solution } \\
\text { wt \% PVP (mol weight) }\end{array}$ & $\begin{array}{l}\text { Coagulation bath } \\
\text { (wt \% NMP) }\end{array}$ \\
\hline A & $5(360,000)$ & 40 \\
B & $75(360,000)$ & 40 \\
C & $10(360,000)$ & 40 \\
D & $10(40,000)$ & 20 \\
E & $10(160,000)$ & 20 \\
F & $10(360,000)$ & 20 \\
\hline
\end{tabular}




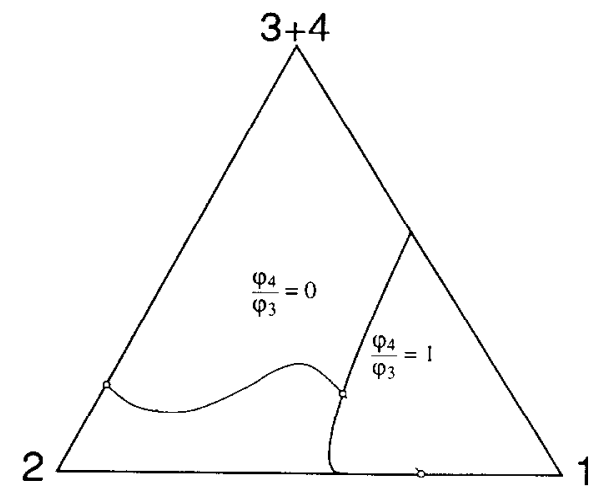

Fig 3 Composition profile for $\phi_{4} / \phi_{3}=10$ Thermodynamic parameters are as in Fig 2 Kinetic parameters are valid for the experimental system water-1-methyl-2-pyrrolidone-poly (ether sulfone)-poly (vinyl pyrrolidone)

$\frac{v_{t} R T}{M_{j} R_{\jmath}}=A \times 10^{-9-B \phi_{1}} \mathrm{~m}^{2} / \mathrm{sec}$

$R_{1 k}=\frac{v_{1}}{v_{2}} R_{2 \mathrm{k}} \quad$ for $k=3,4$

$\imath=1, \jmath=2 \quad A=06, B=00$,

$\imath=3$ or $4, \jmath=2 \quad A=180, B=-4386$

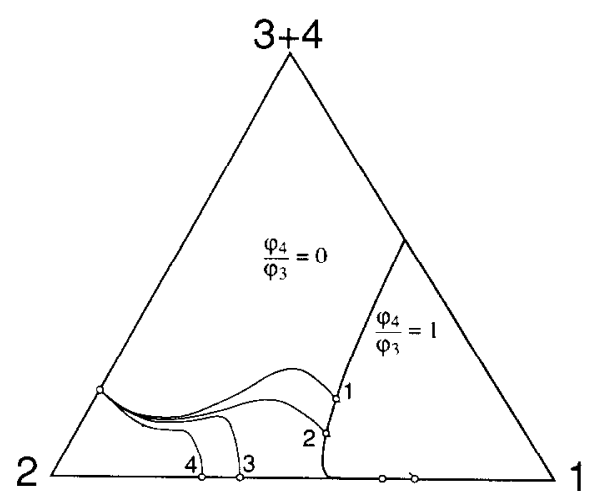

Fig 4 Composition profiles calculated with the same parameters as in Fig 3 The concentration of solvent in the coagulation bath is increasing from 0 to $65 \mathrm{vol} \%$

a high concentration of solvent, the coagulation bath turns slightly turbid, indicatıng dissolution of some polymer in the coagulation bath This is in agreement with the calculated results.

\section{Discussion}

Experiments showed that only an additive of a certain minımum molecular weight and present at a certain minimum concentration is effective to prevent macrovoid formation. We will now discuss how the calculated results may explain these facts

Calculations showed that at all concentrations of solvent in the coagulation bath, delay of demixing is obtained with respect to the phase diagram for the short time scale After some moments of immersion however, the longer time scale, in which polymer-polymer movement is possible, is gaining importance. When the polymeric additive is able to diffuse into the membrane forming polymer lean phase, the 'real' binodal close to the polymer-solvent axis is gaining importance again The area of the demixing gap thereby increases dramatically, and the compositions already created in the top-layer appear to be very unstable. Hence, demixing, once started, will be relatively fast in the top-layer.

Complete equilibrium calculations (1 e without any assumptions concerning concentratıons) show that the p.ymeric additive (4) usually is almost exclusively present in the polymer (3) lean phase The binodal in this case shifts to lower water concentrations in the polymer (3) rich phase than when no additive (4) would have been present in the system. Therefore, as an indication for this situation the binodal valid for a system without any additive present in the system can be used (see Fig. 4)

The change from the 'virtual' binodal for the short time scale to the 'real' binodal that represents real phase equilibria in the phase diagram implies that for solutions containing a polymeric additıve, delay of demixing as described by Reuvers et al [1] cannot occur at all Even at high concentrations of solvent in the coagulation bath (where a solution without 
the additive would exhibit delay of demixing), compositions which are unstable in the long time scale are created in the top-layer during the short time scale From Fig. 4, one might even say, that in the top-layer conditions for spinodal demixing are created. We will expand on this topic in part B

The measurement of light transmission through precipitating membranes indeed ind1cates that addition of a polymeric additıve induces a certain instantaneous demixing process, at conditions that would yield delay of demixing without the additive This can be related to the effects the additive has on macrovold formation, as will be explained in the next paragraph.

\section{Macrovord formation}

Reuvers proposed a mechanism of formation of macrovoids in ternary systems consisting of non-solvent, solvent and membrane forming polymer This mechanism was presented in the preceding part of this work: it will be summarized in short here

A casting solution, demixing according to an instantaneous mechanism, develops higher and higher solvent concentrations in the nuclei of the polymer lean phase present in the proceeding coagulation front. At a certain point, these solvent concentrations become so high locally, that conditions become favorable for delay of demixing (although this is only calculated for a flat geometry, and not for a cylindrical situation as is present here). Delay of demixing is normally characterized, due to loss of solvent which is larger than the influx of non-solvent, by a shrinkage of the polymer solution, and, consequently, an increase in volume of the coagulation bath In this case, a nucleus is serving as a tiny coagulation bath The polymer solution surrounding the nucleus experiences delay of demixing with respect to the nucleus, and the nucleus will grow. As long as the conditions of delayed demixing remain the same, i.e. as long as the solvent concentration in the nucleus remains high enough, the growth of the nucleus continues.

We return to the quaternary system with a polymeric additive As we have seen, in a system with a polymeric additive, delay of demixing does not take place, as long as the two time scales can be operative. Local conditions for delay of demixing cannot take place Growth of a nucleus is effectively blocked by the creation of new nuclei. Apparently, the addition of a polymeric additıve hinders macrovoid formation. This effect can only take place when enough additive is present: at lower concentrations the demixıng gap does not shift enough to the right in the phase diagram to induce any significant effect durıng the short time scale. On the other hand, the diffusion of the two polymers with respect to each other should be slow compared to the diffusion of solvent and non-solvent in the polymer solution Otherwise, it is not possible to distıngush a short time scale from the longer one Delay of demixing will then still be possible To avoıd this, a certain minımum molecular welght of both polymers in the system is required. Since in most cases the molecular weight of the membrane forming polymer is fixed, this means that the molecular weight of the additive should have a certain minimum value This is in agreement with the facts found in the experimental section.

\section{(B) Nodular structures in ultrafiltration membranes}

\section{Theoretical considerations}

Open pore structures in membranes are formed by nucleation and growth of the polymer lean phase in the metastable region between the binodal and the spinodal curve. However the top-layer of ultrafiltration membranes often does not show an open pore structure nor a completely homogeneous gel layer It 

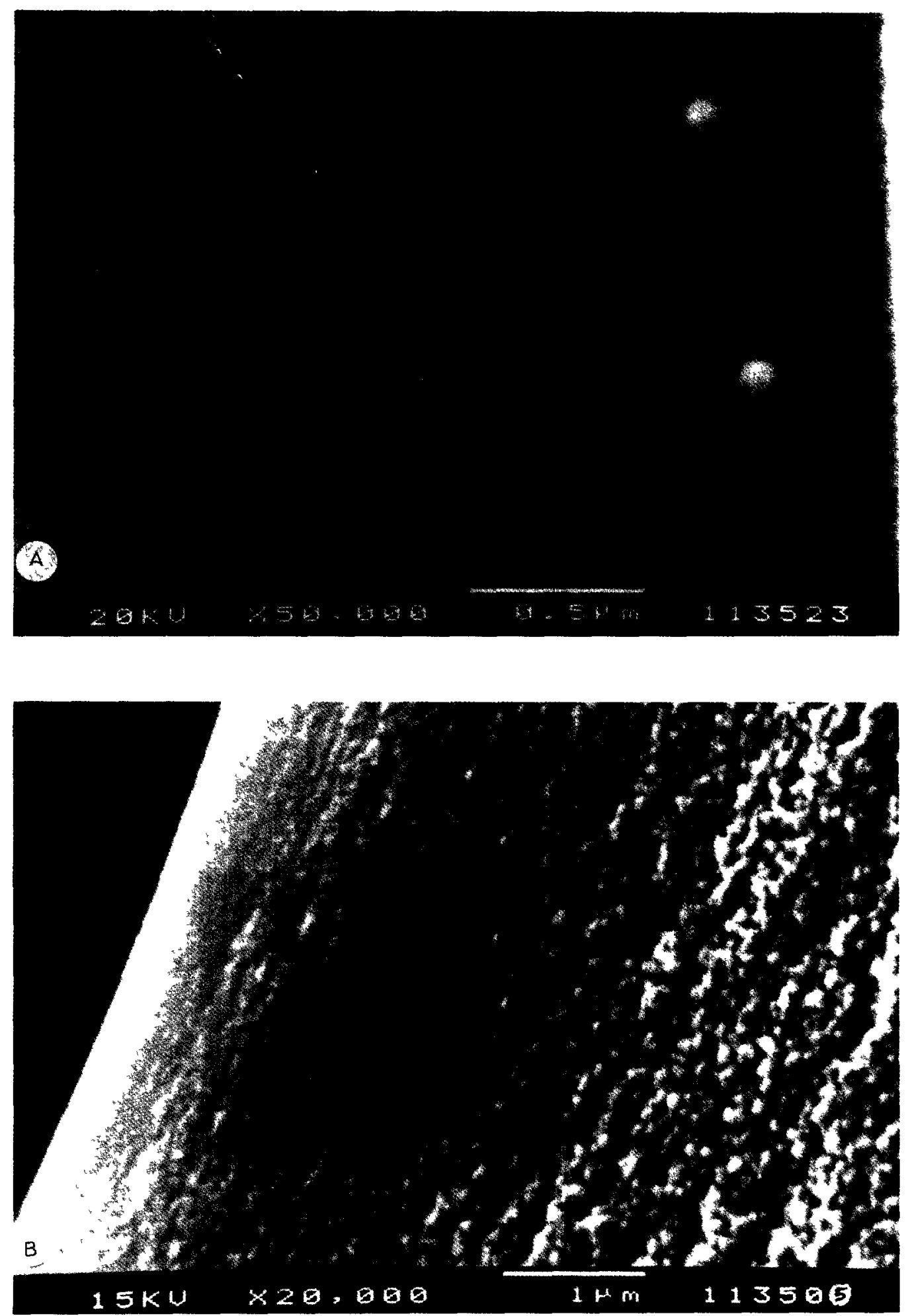
consists of closely packed spheres of polymeric material with a diameter of about $50-200 \mathrm{~nm}$ and is often referred to as a nodular structure [6].

The formation of a nodular structure can not be explained by nucleation of the polymer lean phase It is also not very likely that nucleation of the polymer concentrated phase occurs because this only happens at initially low polymer concentrations, below the critical point A possible explanation for the formation of a nodular structure could be that it is a result of spinodal demixing

\section{Spinodal demixing}

In the spinodal region the homogeneous system is unstable. A nucleus of one of the binodal phases is not necessary to initiate phase separation. A theory of spinodal decomposition has been developed by Cahn [7]. Very small concentration fluctuations with a wavelength above a critical value will grow Due to negative diffusion coefficients "up-hill" diffusion takes place. This means that the amplitude of the wave grows whereas the wavelength remains constant Van Aartsen [8] showed that spinodal demixing can also occur in polymer solutions when quenched to a temperature below the spinodal temperature Combining Cahn's theory of spinodal demixing and Debije's theory describing random concentration fluctuations in polymer solutions an expression for the fastest growing wavelength $\left(D_{\mathrm{m}}\right)$ was found:

$D_{\mathrm{m}}=2 \pi l /\left[3\left(1-T / T_{\mathrm{s}}\right)\right]^{05}$

The wavelength $\left(D_{\mathrm{m}}\right)$ is linearly related to the radius of gyration $(l)$ of the polymer and is therefore dependent on molecular weight as well as on the thermodynamic interaction between polymer and solvent The wavelength decreases if the quenching temperature $(T)$ is lowered, $1 \mathrm{e}$ if the polymer solution is brought deeper inside the spinodal region. The spinodal temperature is dependent on the polymer concentration and is indicated by $T_{\mathrm{s}}$. Smolders et al [9] determined $D_{\mathrm{m}}$ for a 15 weight percent poly-2,6-dimethyl-1,4-phenylene oxide (PPO) solution in caprolactam. The radius of gyration is of the order of $20 \mathrm{~nm}$. At an undercooling of $30^{\circ} \mathrm{C}$ below the spinodal temperature the calculated wavelength is $D_{\mathrm{m}}=260 \mathrm{~nm}$. According to Smolders et al [9] the fast spinodal demixing process is followed by a slower phenomenon to reach the final equilibrium phases At high quenching temperatures this could be measured using dilatometry and electron microscopy By dilatometric measurements it was found that a large change in volume occurring upon quenching is followed by a second volume effect on a longer time scale. Electron microscopy showed droplets of the polymer concentrated phase increasing in size with time. The ultımate structure is fairly uniform with a characteristic distance between the polymer rich regions

\section{Results}

Hollow fibers were spun as described in the experımental set-up of part $A$ of this paper. The membrane morphology of the top-layer is shown in Fig. 5. The surface top view (Fig. 5a) shows a bi-continuous structure which is typical for spinodal decomposition Nodule size is estimated to be ca $50 \mathrm{~nm}$. In the cross section ( $F_{1} g \mathrm{bb}$ ) the structure at the surface is not very well visible Nodular size increases from $50 \mathrm{~nm}$ (at $0.5 \mu \mathrm{m}$ distance from the surface) to $100 \mathrm{~nm}$ ( $5 \mu \mathrm{m}$ below the surface). Below the top-layers

Fig 5 Scanning electron microscopic pictures of hollow fibers (A) surface view, (B) cross section at the outside top-layer region Concentration of poly (ether sulfone) was $17 \mathrm{wt} \%$, concentration of poly (vinyl pyrrolidone) (MW 360,000) was 13 wt \% After an air-gap of $15 \mathrm{~cm}$, the fiber was coagulated in a pure water bath of $26^{\circ} \mathrm{C}$ 
which are shown, the membranes have an open pore structure (not shown in this picture)

In Fig. 6 the cross section of a top-layer for moderate coagulation conditions is shown The coagulation bath contained $60 \%$ of the solvent in the non-solvent Therefore the non-solvent concentration gradient in the top-layer was much smaller. For this membrane nodule size is almost equal over the top-layer thickness. At the surface the nodules are more closely packed.

\section{Discussion}

The nodular structure in ultrafiltration membranes formed by immersion precipitation resembles the structure obtained by quenching a polymer solution This makes spinodal decomposition a reasonable mechanism for nodule formation However, spinodal demixing can not be related to the membrane formation model of Reuvers et al [1] The calculated composition paths can never cross the spinodal curve, because at these compositions the chemical potential gradients and fluxes are all zero If we assume that for the extremely fast formation of ultrafiltration top-layers the established theory based on equilibrium thermodynamics is not valid for the three (or four) component system, an alternative mechanism can be proposed

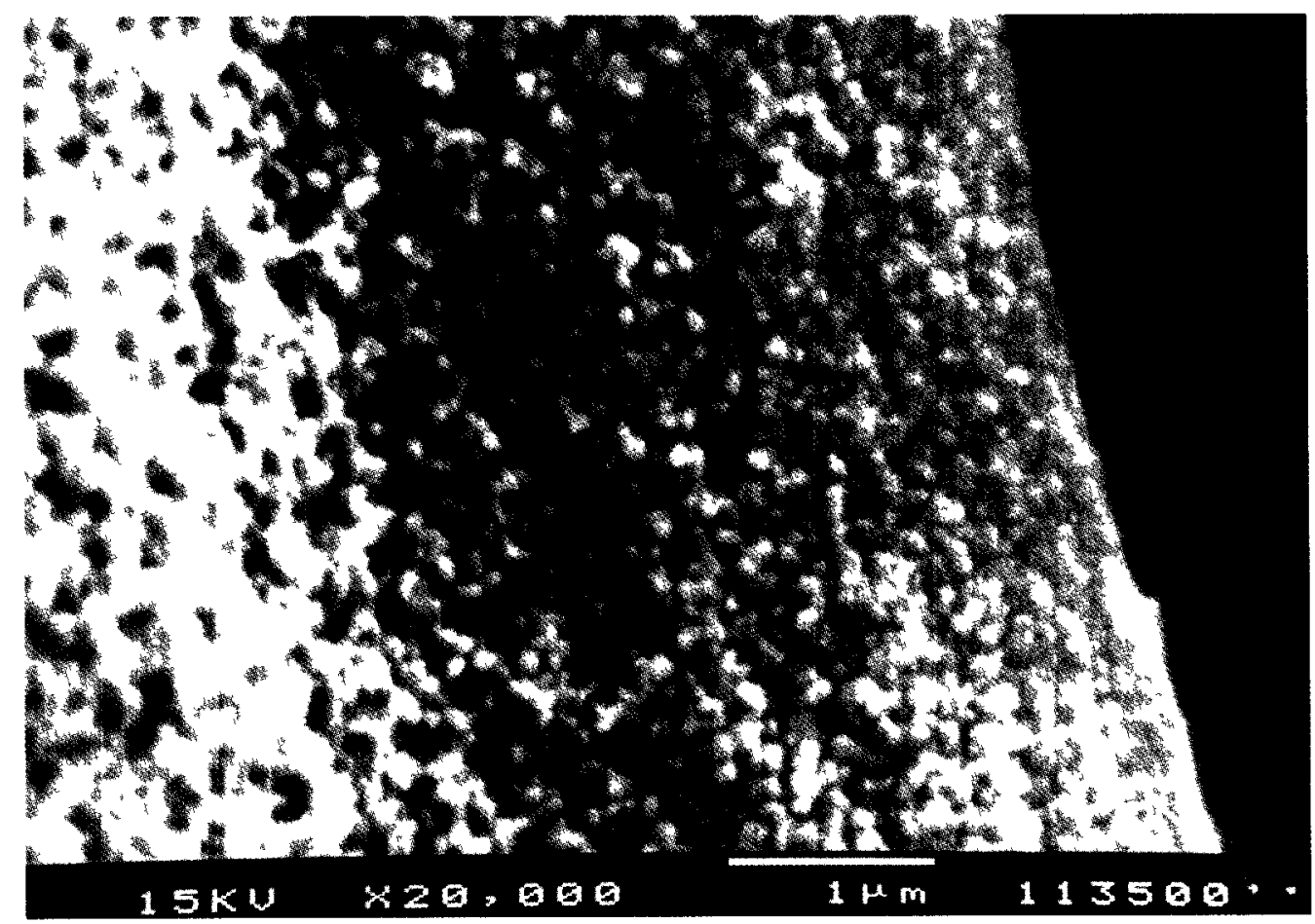

Fig 6 Scanning electron microscopic picture of hollow fibers, cross section at the bore side top-layer Concentration of poly (ether sulfone) was $17 \mathrm{wt} \%$, concentration of poly (vinyl pyrrolidone) (MW 360,000) was 13 wt \% Internal coagulation bath was $60 \mathrm{wt} \%$ NMP in water 


\section{Formation of ultrafiltration top-layers}

In an ultrafiltration membrane-forming system the interaction between solvent and nonsolvent is very high. Upon immersion in the coagulation bath fast exchange of solvent and non-solvent takes place in the top-layer Due to their high molecular weight the polymer molecules are not mobile enough in the initial stage, there is a certain relaxation time Solvent and non-solvent molecules can diffuse through the polymer colls by movement of polymer chain segments while at the same time the polymer chains as a whole do not move at all. When the polymer concentration is kept constant the composition of the polymer solution rapidly crosses the metastable region without being able to demix according to a nucleation mechanism. During this diffusion process only polymer chain segments are moving Therefore the spinodal curve, calculated for a system where polymer molecules as a whole can diffuse, is not valid This means that the composition of the film can enter the region which is the spinodal region in equilibrium conditıons.

Fast penetration into the binodal region without nucleation has also been reported by other authors Accordıng to Kimmerle [10] a certain period is necessary for nucleation to start This induction time becomes smaller according to Kimmerle as the composition changes towards the spinodal curve. So, if diffusion processes are very fast the composition deeply enters the binodal region before phase separation sets in. Cohen et al [11] report that phase separation in viscous polymer solutions is relatively slow compared to the diffusional equilibration of solvent and non-solvent According to them phase separation begins at compositions lyıng at the limit of metastability, i.e. the spinodal curve.

Thus, until the relaxation time of the polymer in solution has elapsed the solution is still homogeneous while the composition has reached the spinodal region When after a cer- tain time the polymer molecules start to diffuse spinodal decomposition occurs rapidly

A difference in mobility between polymer chain segments and polymer molecules as a whole was also distinguished by Tkacik and Zeman [5] They applied quası-elastıc light scattering (QELS) to water-NMP-PES systems For intertwined polymer solutions two clearly distinguishable relaxation times and diffusion coefficients were measured. Relaxation times of a 20 weight percent solution of PES in NMP were $1 \times 10^{-4} \mathrm{sec}$ for the fast mode and $1 \mathrm{sec}$ for the slow mode. Binary diffusion coefficients of a 30 weight percent solution of $\mathrm{PES}$ in NMP were $66 \times 10^{-11} \mathrm{~m}^{2} / \mathrm{sec}$ for the fast mode and $7.5 \times 10^{-16} \mathrm{~m}^{2} / \mathrm{sec}$ for the slow mode, respectively With these values an estimation can be made of the part of the film which will be in the spinodal region at the moment the polymer molecules start to diffuse, $1 \mathrm{sec}$ after immersıon. We use Fick's second law for infinite media [12]

$\frac{C_{x}-C_{1}}{C_{0}-C_{1}}=\operatorname{erf} \frac{x}{2 \sqrt{(D t)}}$

and take the non-solvent concentration at the surface $C_{0}=50 \%$, the initial non-solvent concentration in the polymer solution $C_{1}=0 \%$, and its concentration at the spinodal $C_{x}=20 \%$ The fast mode binary diffusion coefficient for solvent and polymer as given by Zeman and Tkacik [5] is used as the diffusion coefficient for non-solvent through the polymer network The relaxation time of the network is $t=1 \mathrm{sec}$. Then the penetration depth can be calculated as $x=6$ $\mu \mathrm{m}$ This means that a layer of $6 \mu \mathrm{m}$ will be in the spinodal region and can give a nodular structure. This is in agreement with the experimental results.

The wavelength of the fastest growing wave is dependent on the penetration depth into the spinodal region The composition at the surface is the first to cross the spinodal and therefore will have proceeded further into the spi- 
nodal region Here the wavelength will be smaller In Fig. 5 it can be seen that indeed the nodular size is smallest at the surface and becomes larger going deeper into the membrane top-layer.

\section{Quaternary systems}

For a quaternary system with two high molecular weight components other conditions may also give rise to spinodal decomposition. In the previous part is has been described that the composition of the polymer solution can enter the spinodal region as long as the relaxation time of the polymer molecules has not elapsed. But the model, proposed in Part A, for demixıng of quaternary systems can explain the occurrence of spinodal demixing even after the relaxation time has elapsed.

For moderate coagulation conditions, e.g if the coagulation bath contains a considerable amount of solvent, the diffusion processes are slower. As stated in Part A on the short time scale the two polymers can be regarded as one and the relevant binodal is situated on the right hand side of the phase diagram close to the nonsolvent corner. Before this binodal is reached the relaxatıon time has elapsed and the two polymers can diffuse but not with respect to each other Once the two polymers can diffuse with respect to each other, the system gradually starts to react in agreement with the original quaternary binodal and spinodal curve situated more to the left in the phase diagram (see Fig. 3). From this moment on the compositions in the top-layer lie in the unstable region and spinodal demixing occurs rapidly, finally resulting in a nodular structure

In Fig. 6 a nodular structure was formed with a coagulation bath that contained $60 \%$ solvent Due to the smaller concentration difference the coagulation velocity is smaller and normally an open pore structure would be formed. However due to the addition of a second polymer phase separation is faster and a nodular structure is formed

\section{Conclusions from $A$ and $B$}

\section{Macrovold formation}

It appears that the macromolecular nature of the additive is responsible for the suppression of formation of macrovoids The reason is that the two polymers present in the same solution have to diffuse with respect to each other in order to phase separate Since this process, for high enough molecular weight components, is much slower than low-molecular weight diffusion, two time scales are created.

The short time scale is responsible for the creation of a top-layer which has a high nonsolvent content without demixing. These compositions are highly unstable in the longer time scale. Delay of demixing in such a system is not possible anymore Because the formation of macrovords is closely connected to the phenomenon of delayed demixing, macrovord formation is effectively hindered.

\section{Nodular structures}

It has been shown that the nodular structure appearing in the top-layer of ultrafiltration membranes is probably a result of spinodal demixing Although the phase separation process is diffusion controlled there are two ways in which the composition of the polymer solution can cross the spinodal curve

(1) In the first period of fast immersion processes solvent and non-solvent diffuse trough a "fixed" polymer network Thermodynamics based on equilıbrium states are not valid for these first moments after immersion Compositions are reached which appear to lie in the spinodal region when polymer molecules become mobile and the thermodynamics are valid again. 
(11) For systems with a high molecular weight additive, in the first moments after immersion the binodal and spinodal curve are situated close to the non-solvent corner Upon regaining the normal thermodynamic conditions (for the longer time scale) the compositions are found to lie definitely in the spinodal region of the real phase diagram.

\section{List of symbols}

$C_{x} \quad$ concentration at place $x\left(\mathrm{~kg}-\mathrm{m}^{-3}\right)$

$C_{0} \quad$ concentration at the surface $\left(\mathrm{kg}-\mathrm{m}^{-3}\right)$

$C_{1} \quad$ initial concentration in the film (kg$\mathrm{m}^{-3}$ )

$c_{\imath} \quad$ concentration of component $\imath(\mathrm{kg}-$ $\mathrm{m}^{-3}$ )

$D_{\imath \jmath} \quad$ binary diffusion coefficient of components $l$ and $J\left(\mathrm{~m}^{2}\right)$

$D_{\mathrm{m}} \quad$ perturbation wavelength $(\mathrm{m})$

$\Delta G_{\mathrm{m}} \quad$ the enthalpy of mixing (J)

$g_{t j}$ concentration dependent Flory-Huggins interaction parameter between component $\iota$ and $J(-)$

$L_{\imath j} \quad$ diffusivity between components $\imath$ and $J$; to be evaluated with the StefanMaxwell approach

$l \quad$ radius of gyration $(\mathrm{m})$

$m$ modified spatial coordinate in the polymer solution;

$m=\int_{\xi=0}^{\xi=x} \phi_{3} \mathrm{~d} \xi(\mathrm{m})$

$n_{\imath} \quad$ number of moles of component $\imath$ (moles)

$R \quad$ gas constant ( $\left.\mathrm{J}-\mathrm{mole}^{-1}-\mathrm{K}^{-1}\right)$

$R_{\imath} \quad$ Stefan-Maxwell friction coefficient between components $\imath$ and $l$, found from binary diffusion data [1]

$T$ temperature (K)

$T_{\mathrm{s}} \quad$ spinodal temperature (K)

$t \quad$ time (sec)

$v_{l} \quad$ specific volume of component $\imath\left(\mathrm{m}^{3}\right.$ $\mathrm{kg}^{-1}$ )

$\bar{v}_{\imath} \quad$ velocity of component $\iota\left(\mathrm{m}-\sec ^{-1}\right)$ spatial coordinate originatıng from the interface, going into the polymer solution $(\mathrm{m})$

$y$ spatial coordinate originating from the interface, going into the coagulation bath $(\mathrm{m})$

$\mu_{i} \quad$ chemical potential of mixing of component $l \quad$ (equal to $\left.\partial \Delta G_{\mathrm{m}} / \partial n_{\imath}\right) \quad(\mathrm{J}-$ mole $^{-1}$ )

$\phi_{\iota} \quad$ volume fraction of component $\iota(-)$

$\chi_{\imath j} \quad$ concentration independent FloryHuggins interaction parameter between component $\iota$ and $J(-)$

\section{References}

1 A J Reuvers, J W A van den Berg and C A Smolders, Formation of membranes by means of immersion precipitation Part I A model to describe mass transfer durıng ımmersion precipitation, J Membrane Scı , 34 (1987) 45

A J Reuvers and C A Smolders, Formation of membranes by means of immersion precipitation Part II The mechanism of formation of membranes prepared from the system cellulos acetate-acetone water, J Membrane Sc1 , 34 (1987) 67

2 L Yilmaz, A J McHugh, Modelling of asymmetric membrane formation I Critique of evaporation models and development of a diffusion equation formalısm for the quench period, J Membrane Scı, 28 (1986) 287

3 I Cabasso, E Klein and J K Smith, Polysulphone hollow fibers II Morphology,J Appl Polym Sc1 , 21 (1977) 165

P Aptel, N Abıdıne, F Ivaldı and J P LaFalle, Polysulphone hollow fibers - Effect of spmning conditions on ultrafiltration properties, J Membrane Scı, 22 (1985) 199

L Y Lafrenière, F D F Talbot, $T$ Matsuura and S Sourirajan, Effect of poly (vinyl pyrrolidone) additive on the performance of polyethersulphone ultrafiltration membranes, Ind Eng Chem, Res, 26 (1987) 2385

T A Tweddle, O Kutowy, W L Thayer and S Sourirajan, Polysulphone ultrafiltratıon membranes, Ind Eng Chem, Prod Res Dev , 22 (1983) 320

4 F Altena and C A Smolders, Calculation of a liquidliquid phase separation in a ternary system of a polymer in a mixture of a solvent and a nonsolvent, Macromolecules, 15 (1982) 1491 
5 L Zeman and G Tkacik, Thermodynamic analysis of a membrane formung system Water/N-methyl-2-pyrolddone/polyethersulfone, J Membrane $\mathrm{Sc1}, 36$ (1988) 119

G Tkacık and L Zeman, Component mobility analysis in the membrane forming system water $/ \mathrm{N}$ methyl-2-pyrrolıdone/polyethersulphone, J Membrane Sc1 , 31 (1987) 273

6 L Broens, F W Altena and C A Smolders, Asymmetric membrane formation as a result of phase separation phenomena, Desalınatıon, 32 (1980) 33

7 J W Cahn, Phase separation by spmodal decomposition in isotropic systems, J Chem Phys, 42 (1965) 93

8 J J van Aartsen, Light scattering of polymer solu- tıons durıng lıquid-lıquid phase separatıon, Eur Polym J , 6 (1970) 919

9 C A Smolders, J J van Aartsen and A Steenbergen, Liquid-liquid phase separation in concentrated solutions of non-crystallizable polymers by means of sp1nodal decomposition, Kolloıd-Z u Z Polym, 243 (1971) 14

10 K Kımmerle, Quantitative Betrachtung des Phaseninversionsprozesses bei der Herstellung von Membranen, $\mathrm{PhD}$-thesis, Stuttgart, 1988

11 C Cohen, G B Tanny and S Prager, Diffusion-controlled formation of porous structures in ternary polmer systems, J Polym Scı , 17 (1979) 477

12 J Crank, The Mathematics of Diffusion, 2nd edn Clarendon Press, Oxford, 1975 